\title{
Numerical Computational Study Applied to Natural and Forced Convection in Cylindrical and Semi-Circular Thermal Tanks
}

\author{
Darci Luiz Savicki \\ Federal University of Rio Grande -Institute of Mathematics, Statistics and Physics \\ Av. Itália Km 8, CEP 96201-900, Rio Grande, RS, Brazil \\ E-mail: darcisavicki@furg.br
}

\begin{abstract}
In many applications cylindrical tanks with large aspect ratio are used. In cases where the diameter is limited in project and the ratio length - diameter is more than 2.75, replacement of the conventional cylindrical tank for a semi-circular one showed economical advantages by reducing the ratio surface area/volume. In this work, cylindrical and semicircular tanks were numerically studied to investigate the temperature and velocity fields in tanks subject to natural and forced convection. The numerical results obtained in this study showed that, in cases where the aspect ratio of the cylindrical tank is high (> 2.75), the semicircular tanks were more advantageous than the usual cylindrical shape.
\end{abstract}

\section{Introduction}

One of the issues of interest in natural convection is the optimization of thermal energy storage equipment. An illustrative case is solar water heating systems, in which a good performance of the storage tank represents a considerable increase in the global efficiency. In such systems, one of the most desirable characteristics is an effective thermal stratification. The thermal stratification inside the tank depends on the velocity of the inlet jet as well as on the difference between the inlet and outlet temperatures of the collectors. In this work natural and forced convection in cylindrical and semi-circular tanks are numerically studied, using numerical simulation.

\section{Physical and Mathematical Model}

\subsection{Some mathematical relationships between the tanks under study}

In solar water heating systems, cylindrical tanks commonly have a much greater length than the diameter, as can be seen in [3], [4]. The aspect ratio ( $r=L / D)$ of such tanks ranges from 2 to 5. In solar systems powered by thermosyphon, horizontal cylindrical tanks, with diameters exceeding $0.7 \mathrm{~m}$, are used because the available space for accommodation below the roof is very limited, where a horizontal cylindrical tank is used for hot water storage. Therefore, in order to store a sufficient volume of water, these tanks may be longer than $3 \mathrm{~m}$ and have aspect ratio of about 5. An alternative to avoiding too long tanks which has been little explored so far is the replacement of cylindrical geometry for another such as a semi-circular one, as shown in Figure 1. Semi-circular tanks have some attractive characteristics, such as:

1- They allow storing the same volume as the conventional cylindrical tank, but using half-length (same height);

2- In cases where the conventional cylindrical tank shows high aspect ratio (above 2.75), they allow reducing cost and heat losses, due to the reduction of the heat exchange area;

3- They are easy to be installed due to the flat bottom.

As a generic example, a cylindrical tank of $\mathrm{r}$ and $\mathrm{z}$ dimensions and a semi-circular tank of $\mathrm{R}=2 \mathrm{r}$ and $\mathrm{Z}$ dimensions are taken into account, as shown in Figure 1. For semi-circular tank to have the same external area as the cylindrical tank, its length should be

$$
Z=\frac{\pi(z-r)}{\pi+2}
$$


And, in order to have the same volume as the cylindrical tank, semi-circular tank must have length of

$$
Z=\frac{z}{2}
$$

Equating (1) and (2) and evidencing the variable $z$, the following is obtained:

$$
z=\frac{2 \pi r}{\pi-2}
$$

Finally, the maximum aspect ratio to make the tank cylindrical with a smaller surface area (when compared to semi-circular tank) is found to be

$$
\text { ar }=\frac{\pi}{\pi-2} \approx 2.752
$$

For example, for a cylindrical tank with radius $0.35 \mathrm{~m}$ and length $3.6 \mathrm{~m}$, a corresponding semi-circular tank with $\mathrm{R}=0.7 \mathrm{~m}$ and $\mathrm{Z}=1.8 \mathrm{~m}$ would store the same volume, but with external area reduced about $8 \%$. Thus, it is estimated that semi-circular tanks would have a reduction in cost and heat loss.

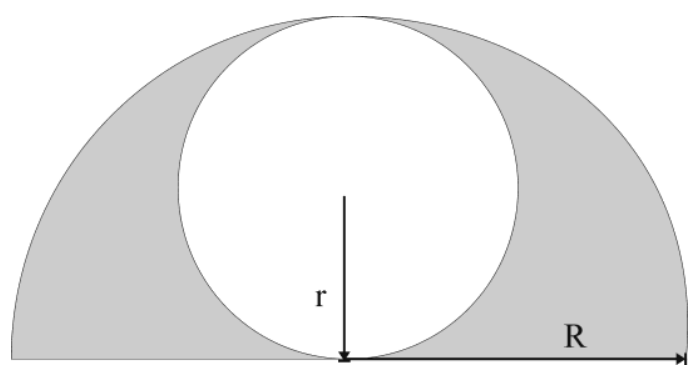

Figure 1. Cross section for cylindrical (inscribed) and semi-circular tanks.

For the numerical simulation two tanks were used, with the same internal volume and the same insulation thickness. The cylindrical tank has internal radius and length of $r=0.25 \mathrm{~m}$ and $\mathrm{z}=1.5 \mathrm{~m}$, respectively. As thermal insulation, $0.02 \mathrm{~m}$ of expanded polystyrene is used. The semi-circular tank has internal radius and length of $\mathrm{R}=0.5 \mathrm{~m}$ and $\mathrm{Z}=0.75 \mathrm{~m}$, respectively. With these dimensions, the external area of the semi-circular tank is approximately $1 \%$ less than the cylindrical one. It is assumed that both tanks are found at a room temperature of $20^{\circ} \mathrm{C}$ and the heat transfer coefficient is $8 \mathrm{~W} / \mathrm{m}^{2}{ }^{\circ} \mathrm{C}$. The water temperature in the tanks at the beginning of the cooling process is $60^{\circ} \mathrm{C}$. In the simulation of hot water consumption, the replacement water temperature was considered at $20^{\circ} \mathrm{C}$.

Figure 3 and Figure 4 give a view of the computational domain of both tanks, indicating the position of the jet inlet and outlet.

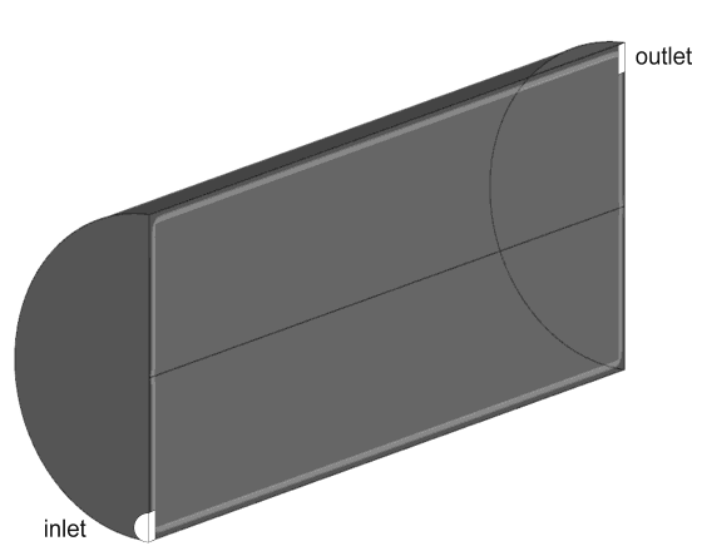

Figure 2. Domain calculation for cylindrical tank.

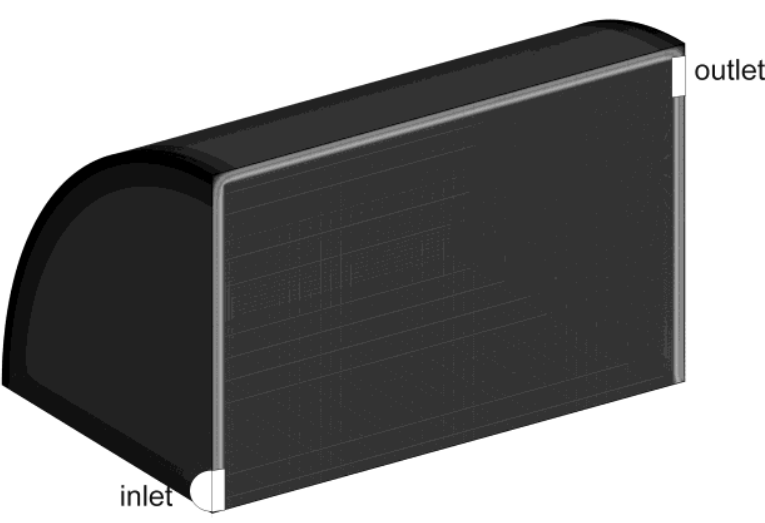

Figure 3. Domain calculation for semi-circular tank. 
For the determination of the temperature and velocity fields inside the cylindrical horizontal tank, it was necessary to solve the energy and momentum equations in three directions: radial, angular, and axial. Details of the mathematical model and numerical solution can be seen in [1] and here will be omitted for brevity.

For simulations of the cooling process, the calculation domain was just $1 / 4$ of the physical body, assuming two symmetry planes and for simulations with charge and discharge, the calculation domain was just $1 / 2$ of the physical body, considering the symmetry in the plane through the vertical diameter. The inlet and outlet duct diameters are $0.0254 \mathrm{~m}$.

\section{Numerical Results}

\subsection{Simulation of hot water consumption}

This study included the two tanks mentioned previously subject to water inlet and outlet in order to measure the hot water consumption by the top and replacing this volume with cold water through the base. The cold water jet enters the bottom of the tank while hot water is extracted (consumption) by the top at a rate of 8 liters per minute. At the initial instant, the water inside the tank is considered to be evenly distributed at $60^{\circ} \mathrm{C}$. The physical time simulated was 10 minutes. The tests of mesh independence and time step were carried out as shown in Tables 1 and 2.

\begin{tabular}{|c|c|c|c|c|c|}
\hline Cylinder & Mesh 1 & Mesh 2 & Mesh 3 & Mesh 4 & Mesh 5 \\
\hline Points in $(r, \theta, z)$ & $(25,79,75)$ & $(35,105,100)$ & $(40,126,119)$ & $(45,140,134)$ & $(49,153,145)$ \\
\hline Time step & 0.5 & 0.1 & 0.05 & 0.05 & 0.05 \\
\hline Total points & 148125 & 367500 & 599760 & 844200 & 1087065 \\
\hline
\end{tabular}

Table 1. Meshes for cylindrical tank

\begin{tabular}{|c|c|c|c|c|c|}
\hline Semi-circular tank & Mesh 1 & Mesh 2 & Mesh 3 & Mesh 4 & Mesh 5 \\
\hline Points in $(r, \theta, z)$ & $(50,79,38)$ & $(67,105,50)$ & $(81,126,62)$ & $(91,142,69)$ & $(98,153,75)$ \\
\hline Time step & 0.5 & 0.1 & 0.05 & 0.05 & 0.05 \\
\hline Total points & 150100 & 351750 & 632772 & 891618 & 1124550 \\
\hline
\end{tabular}

Table 2. Meshes for semi-circular tank

An additional study showed that the mesh independence is achieved through the mesh 3. For this mesh, Figures 4 and 5 show slices of the distribution of temperature and velocity fields for both tanks.

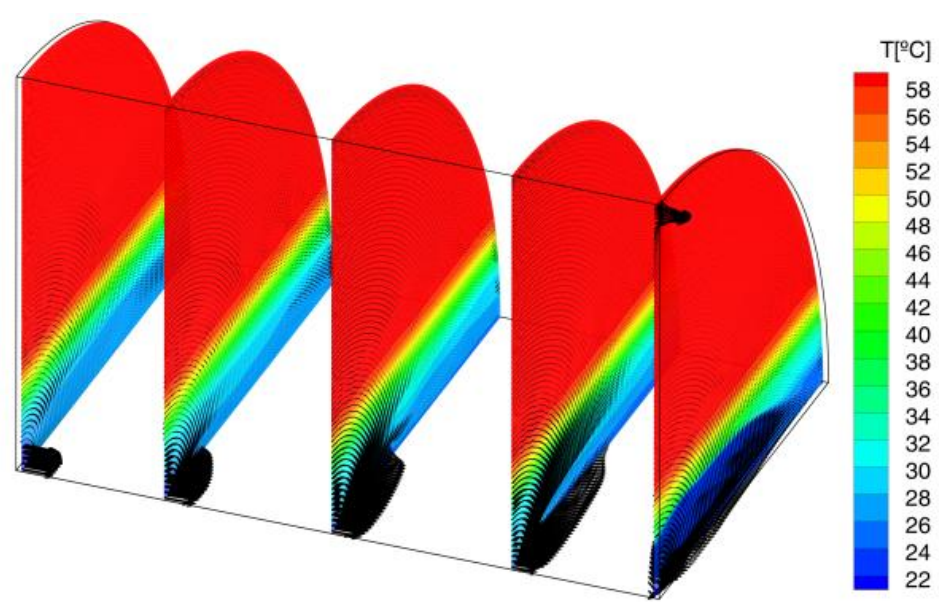

Figure 4. Slices in temperature field for semi-circular tank. 


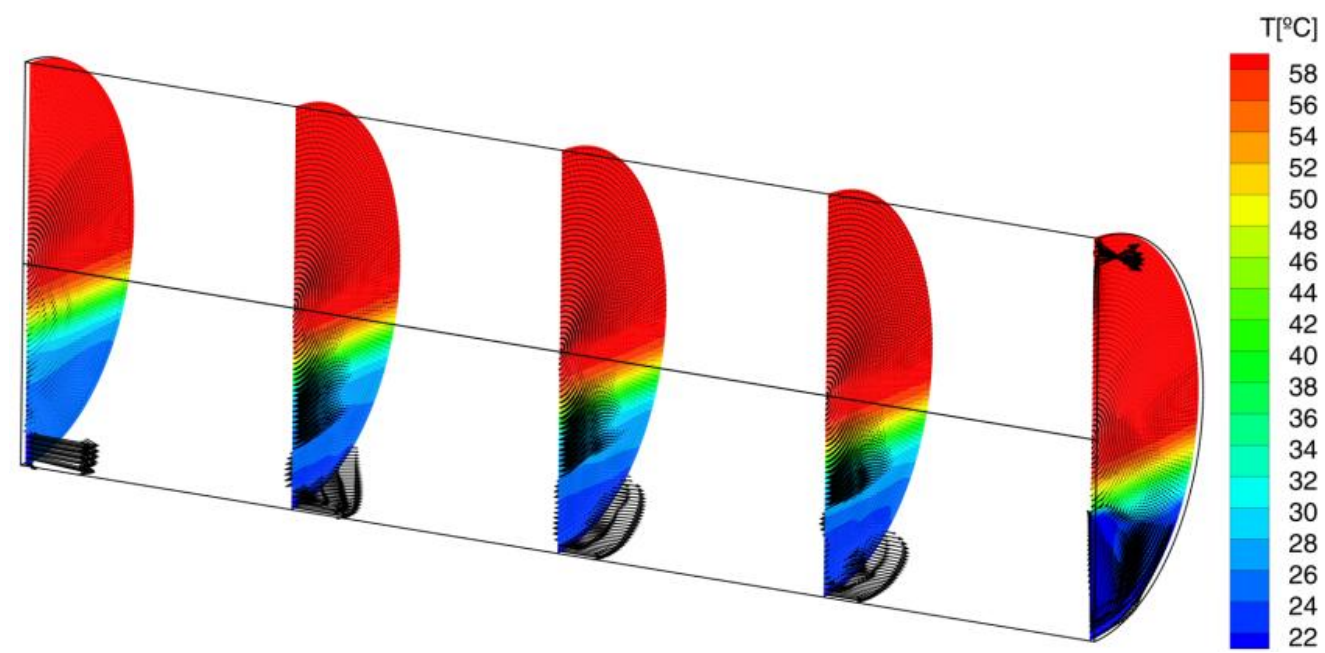

Figure 5. Slices in temperature field for cylindrical tank.

There was a strong thermal stratification in both reservoirs. However, there are important differences to be noticed. First, the semi-circular tank showed a more scattered inlet jet, because its bottom is wide and flat. This feature may be particularly important in applications where it is desired to reduce the speed of the inlet jet and thereby reduce turbulence. Second, the semi-circular tank has a larger wetted area bathed with cold water. It means that at the bottom of the semi-circular tank the heat transfer is lesser than that of the corresponding area in the cylindrical tank. In other words, there is a reduced heat loss. If the cooling time is long, this effect can be significant.

\subsection{Simulation of natural convection}

In order to study the behavior of the two tanks subject only to heat loss by natural convection, mesh 3 for both tanks respectively was conducted to numerical simulation for a 10 hour cooling. A case with $2 \mathrm{~cm}$ of thermal insulation thickness was simulated. Figures 6 and 7 show the temperature field inside the tanks at $10 \mathrm{~h}$ cooling.

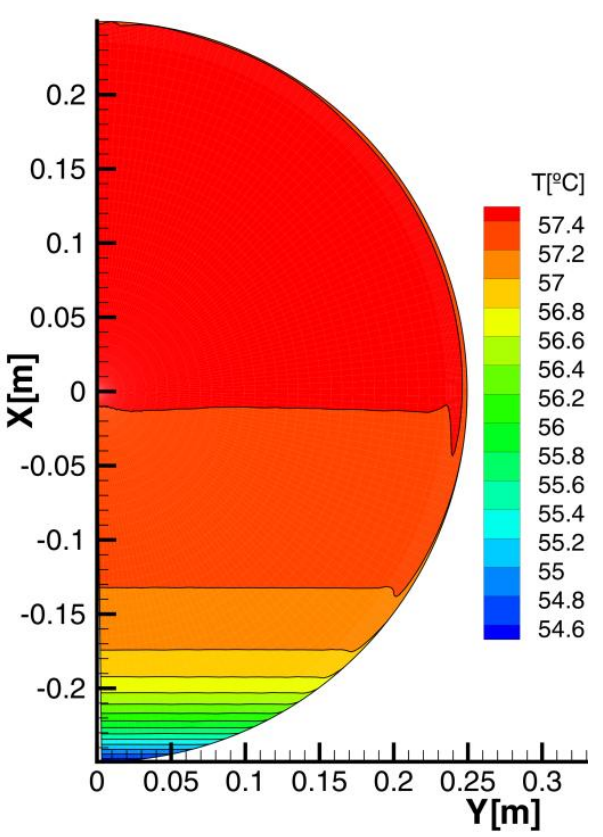

Figure 6. Temperature field in a cross section in the middle of cylindrical tank.

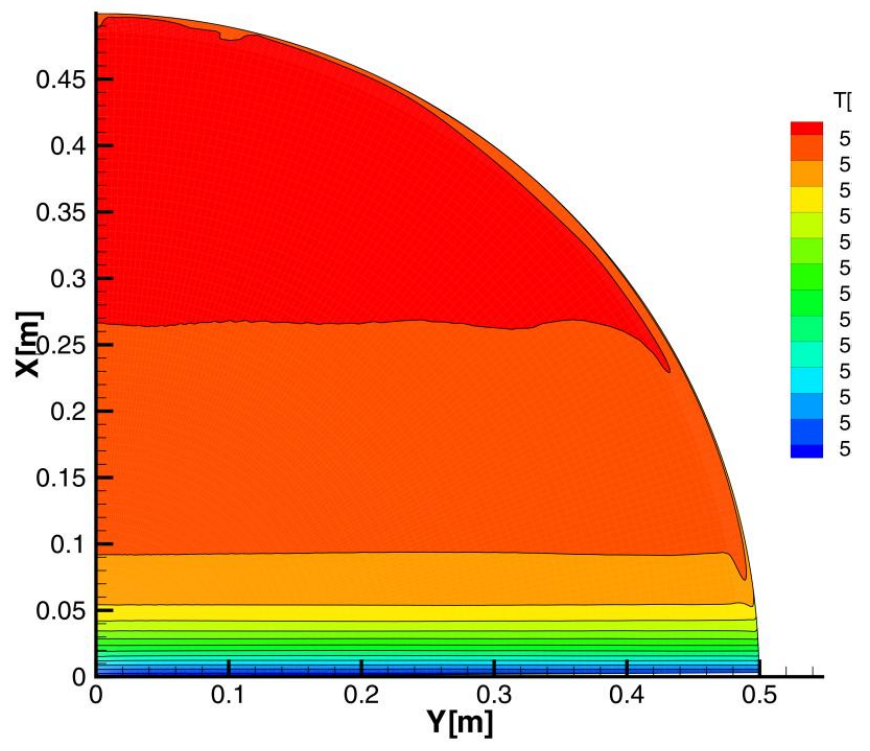

Figure 7. Temperature field in a cross section in the middle of semi-circular tank. 
As shown in Figures 6 and 7, there were similar vertical profiles of thermal stratification. However, in semi-circular tank, the coldest water at the bottom of the tank is deposited on the widest layer because of the geometry of the tank. The evolution of stratification process was similar in both tanks. After an initial time step, the temperature field becomes stratified and subsequently there is basically a vertical displacement of the temperature profile.

Figure 8 shows a comparison of temperature profiles after $10 \mathrm{~h}$ of cooling.

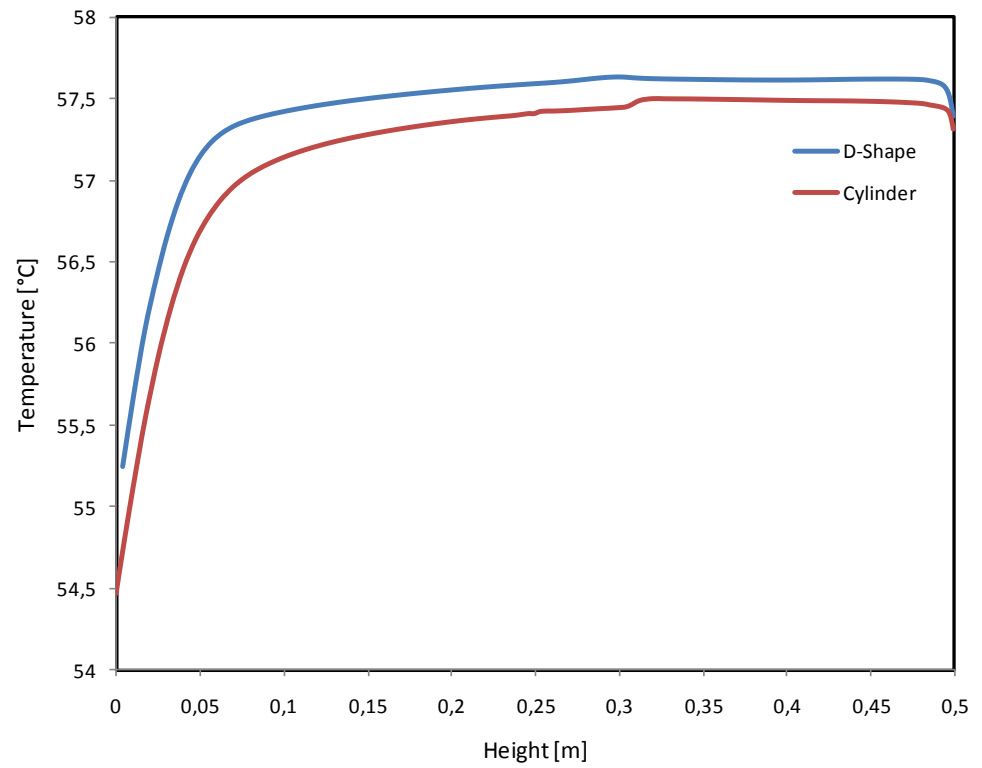

Figure 8. Temperature profiles at 10 hours for cylindrical and semi-circular (D-shape) tanks.

Although there were similar profiles of thermal stratification, in semi-circular tanks temperatures are slightly higher than in cylindrical ones, showing a lower heat loss. As explained earlier, this difference should increase when the comparison is made using cylindrical tanks with greater aspect ratio, such as 4 or $5 \mathrm{~m}$, for example.

Figure 9 shows the pattern of mean temperature throughout $10 \mathrm{~h}$ of simulation.

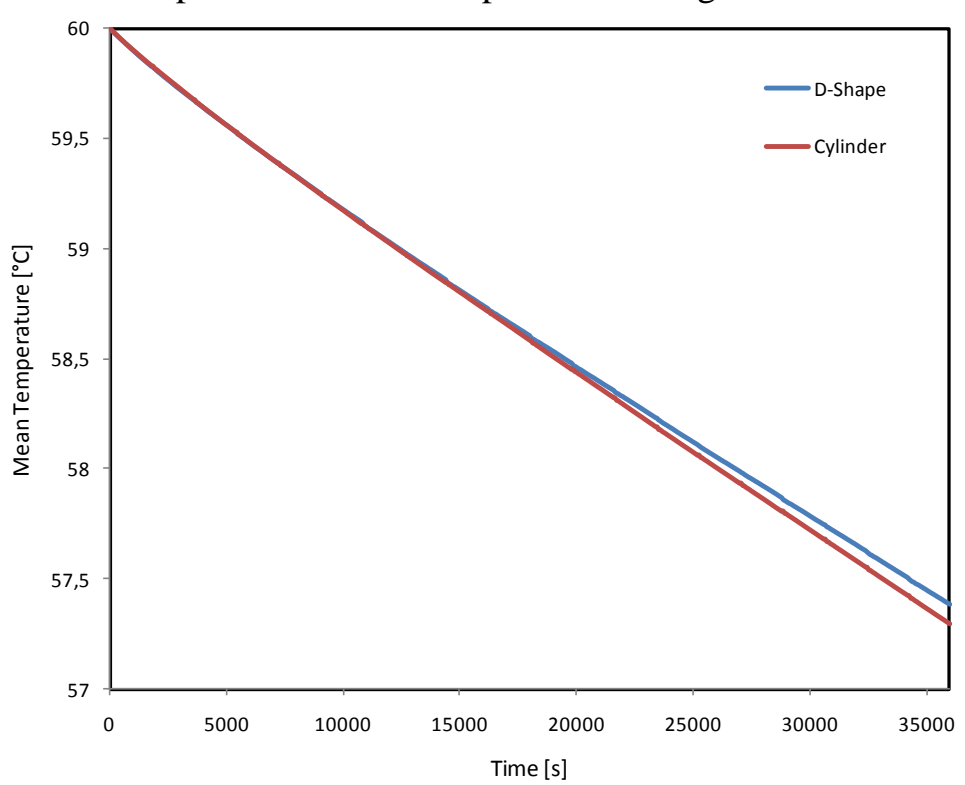

Figure 9. Mean temperature for cylindrical and semi-circular (D-shape) tanks. 
For the time range (10 hour) considered, the reduction of temperature is approximately linear and shows that between this two tank, the variation in mean temperature is small (approximately 0.2 degrees). Also, as observed previously, in semi-circular tanks heat loss was slightly lower than in cylindrical ones.

\section{Conclusions}

This study carried out a numerical study of thermal and hydrodynamics behavior of cylindrical and semi-circular tanks was carried out. Two cases were considered, one of hot water consumption and another of natural convection cooling. The results for simulation of hot water consumption showed that semi-circular tanks reduce the inlet jet speed and thereby reduce turbulence and mixture. Also, semi-circular tanks have a larger wetted area bathed with cold water at the bottom, so that the heat transfer is lower than in cylindrical tanks. That is, there is a reduced heat loss.

For the problem of natural convection cooling, the results showed that the profile of thermal stratification in semi-circular tanks is similar to the cylindrical ones, and that semicircular tanks cool a bit more slowly than cylindrical ones. However, the conclusions herein presented are dependent on the aspect ratio of the tanks. Therefore, further studies are made necessary with other aspect ratios of tanks. In addition, it is important to study the cooling process for both tanks after hot water consumption and replacement of this volume with cold water.

\section{References}

[1] Savicki, D. L.; Vielmo, H. A. ; Krenzinger, A. "Three-dimensional analysis and investigation of the thermal and hydrodynamic behaviors of cylindrical storage tanks". Renewable Energy, v. 36, p. 1364-1373, 2011.

[2] Savicki, D. L.; I. N. Sabin . "Development of a Computational Model Applied to Forced Convection in Cylindrical and D-Shaped Thermal Tanks". COBEM 2011,Natal, RN.

[3] Astrosol, “Boiler Solar”, http://www.astrosol.com.br/boiler_solar.asp, 23 Feb. 2011.

[4] Fibratec, Reservatório Térmico Solar - Boiler, http://www.fibratec.com.br/index.php, 23 Feb. 2011. 